\title{
Olanzapine-valproate combination versus olanzapine or valproate monotherapy in the treatment of bipolar I mania: a randomized controlled study in a Chinese population group
}

\author{
This article was published in the following Dove Press journal: \\ Neuropsychiatric Disease and Treatment \\ 25 May 2015 \\ Number of times this article has been viewed
}

\author{
Lei $\mathrm{Xu}{ }^{\prime}$ \\ Yunrong Lu' \\ Ying Yang' \\ Yanping Zheng' \\ Fang Chen' \\ Zheng Lin $^{2}$ \\ 'Department of Geriatric Diseases, \\ ${ }^{2}$ Department of Psychiatry, Second \\ Affiliated Hospital of Zhejiang \\ University School of Medicine, \\ Hangzhou, People's Republic of China
}

\begin{abstract}
Background: Bipolar disorder (BP) is a mental illness that has a high social burden estimated by disability-adjusted life years. In the present study, we investigated the efficacy of olanzapinevalproate combination therapy versus olanzapine or valproate monotherapy in the treatment of bipolar I mania in a Chinese population group.
\end{abstract}

Subjects and methods: Patients aged 19-58 years who had had an acute manic episode of BP were enrolled in the present study and randomly assigned to receive $600 \mathrm{mg}$ sodium valproate daily (group A), $10 \mathrm{mg}$ olanzapine daily (group B), or a combination of $600 \mathrm{mg}$ olanzapine and $10 \mathrm{mg}$ sodium valproate daily (group C) for 4 weeks. The primary outcome was reduction in Young Mania Rating Scale (YMRS) scores. The secondary outcome was assessed with the Clinical Global Impression - Bipolar (CGI-BP) scale. Adverse reactions, such as weight gain, sleepy, and dizziness were also evaluated. Statistical analysis was carried out on a per-protocol basis.

Results: Patients in groups B and C showed significant improvement in YMRS scores compared with those in group A $(P<0.01)$ during weeks $1-4$ of treatment. Patients in group $\mathrm{C}$ showed significant improvement in YMRS scores compared with those in group $\mathrm{B}(P<0.01)$ only after 4 weeks of treatment. Furthermore, after 3-4 weeks of treatment, patients in groups B and C showed significantly greater improvement in CGI-BP scale scores compared with group A $(P<0.05)$, while Group $\mathrm{C}$ demonstrated significantly greater improvement in CGI-BP scale scores than group B $(P<0.01)$. No significant difference existed in extrapyramidal reactions among these groups. Adverse reactions, including weight gain, drowsiness, dizziness, and constipation, were stronger in groups $\mathrm{B}$ and $\mathrm{C}$ than in group $\mathrm{A}(P<0.05)$.

Conclusion: The combination therapy with olanzapine and sodium valproate had higher efficacy than monotherapy in patients with bipolar mania, which provides a crucial insight of the treatment regimen during clinical practice.

Keywords: combination therapy, monotherapy, Young Mania Rating Scale score, Clinical Global Impression-Bipolar scale

\section{Introduction}

Bipolar disorder (BP) is a mental disease with a high social burden measured by disability-adjusted life years, and the prevalence of BP is approximately $2.0 \%$ of the general population. ${ }^{1,2}$ Traditionally, lithium and sodium valproate were recommended as the first-line medications for severe manic or mixed-phase BP in accordance with the Expert Consensus Guidelines Series set by the American Psychiatric Association. However, these drugs cannot produce rapid sedative effects, and thus failed to manage
Department of Psychiatry, Second Affiliated Hospital of Zhejiang University School of Medicine, 88 Jiefang Road, Hangzhou, Zhejiang 310009, People's Republic of China

Tel +86 57I 87767233

Fax +86 57I 872I 8864

Email linzzr@।26.com 
the acute manic episode of BP. ${ }^{3}$ Therefore, the addition of a mood stabilizer to the conventional antipsychotic therapy would be a useful strategy for the treatment of bipolar I manic episodes. Typical antipsychotics, such as haloperidol and chlorpromazine, produce a series of adverse reactions, such as extrapyramidal side effects, orthostatic hypotension, and liver damage, ${ }^{4}$ which are not tolerated by many patients. In the meantime, atypical antipsychotics, such as risperidone and olanzapine, which have been widely used in the treatment of schizophrenia, ${ }^{5}$ have been credited with good therapeutic effects and rare side effects.

Olanzapine, a novel atypical antipsychotic drug, has been demonstrated in several placebo-controlled trials to possess acute antimanic effects through either monotherapy or combination with other psychotropic agents. ${ }^{6-8}$ Further studies have assessed the antimanic effects of olanzapine in comparison with mood stabilizers, and found that olanzapine had better efficacy than divalproex and lithium., ${ }^{9,10}$ Recently, several trials that added olanzapine to ongoing lithium or valproate therapy also showed positive outcomes. ${ }^{11-13}$ While all these results were from trials for Caucasians, rare studies included Asian populations. Moreover, populations in previous studies were not drug-naïve subjects, which might have skewed the results. The present study aimed to investigate the efficacy and safety of a combined therapy with olanzapine and sodium valproate in the management of acute manic episodes of BP, and compare differences between the combined therapy and olanzapine or sodium valproate monotherapy in a Chinese population.

Through this study, we report that the combination of olanzapine-valproate increases Clinical Global Impression - Bipolar (CGI-BP) scale scores compared with either olanzapine or valproate monotherapy. Our work presents a novel idea of olanzapine-valproate combination therapy versus olanzapine or valproate monotherapy to improve clinical outcome in bipolar I manic episode treatment.

\section{Subjects and methods Subjects}

This study recruited 120 patients with an acute manic episode of BP, who were recruited by the Department of Psychiatry of the Second Affiliated Hospital of Zhejiang University School of Medicine. These patients (60 drug-naïve males and 60 drug-naïve females) were in their first acute manic episode when included in this study. The average age was $30.7 \pm 7.8$ years (range 19-60 years). All of the patients were diagnosed with bipolar I by qualified psychiatrists according to the fourth edition of the Diagnostic and Statistical
Manual of Mental Disorders (DSM-IV). The Young Mania Rating Scale (YMRS) was used to assess the severity of BP. ${ }^{14}$ Patients with a YMRS total score $\geq 17$ were recruited in the study. The exclusion criteria were: 1) female patients with pregnancy or lactation; 2) severe and unstable diseases, including cardiovascular, respiratory, liver, kidney, gastrointestinal, neurological, endocrine, immune, blood-system conditions, narrow-angle glaucoma, and seizures; 3) substance dependence (except tobacco) according to the DSM-IV standards; 4) history of untolerated use of olanzapine or sodium valproate; and 5) history of use of any antipsychotics or mood stabilizers. The study was approved by the Medical Ethics Committee of the Second Affiliated Hospital of Zhejiang University. All subjects had given informed consent prior to inclusion in the study.

\section{Study design}

The study was a prospective double-blind randomized controlled trial. For randomization, a random number table with sequentially numbered, opaque, and sealed envelopes was used to conceal the allocation sequence. Trial medications were administered by nurses. This regimen was continued until the development of severe adverse events or up to 28 days, whichever was sooner. The physician kept the randomization code, and no rater became aware of treatment allocations before requesting unmasking at the end of the study. For group A, patients initially received sodium valproate (Xiangzhong Pharmaceutical Co Ltd, Hunan, People's Republic of China) at $0.6 \mathrm{~g} /$ day (two to three times per day orally). The dose of sodium valproate was gradually increased to $1.2-1.8 \mathrm{~g} /$ day based on the patients' reaction. For group B, patients initially received olanzapine (Eli Lilly and Company, Indianapolis, IN, USA) at $10 \mathrm{mg} /$ day (once a day orally). The dose of olanzapine was adjusted by 5-20 mg/day based on the patients' condition. For group C, patients received both olanzapine and sodium valproate, which were administered in the same manner as in groups A and B. This whole treatment course lasted for 4 weeks, and clinical data were collected at the beginning of the trial and the end of every week by interview. Treatment was stopped when severe side effects occurred or the disease worsened. Aside from trial medications, no other drugs were permitted during the study.

\section{Assessments}

Patient assessments were conducted by a professional psychiatrist who was blind to the experimental condition. The YMRS was used to evaluate the severity of manic symptoms. 
The primary measure of the efficacy of drugs was the mean change from baseline to end point in the YMRS total score. The secondary measure was CGI-BP scale total score. Clinical responses on the YMRS were defined as an improvement of $50 \%$ or greater. ${ }^{3}$ Patients were assessed once a week after treatment.

Any treatment-emergent adverse effects were recorded, were monitored mostly by the subjects and the accompanying family members, and were evaluated by the professional interviewer. Any new adverse reaction emerging with the subjects at least once was regarded as a treatment-emergent adverse effect. Specifically, weight gain was defined as a gain of $5 \%$ or greater, and extrapyramidal reactions were assessed by the Simpson-Angus Scale. Severe adverse effects and poor efficacy were classified as: 1) patients could barely tolerate or accept the medications, and 2) the interviewer concluded that the trial was not beneficial to patients. Patients' body weight, heart rate, and blood pressure were recorded weekly. Laboratory tests, including routine blood test, hepatic function test, blood glucose monitoring, lipid panel screen, and electrocardiograph, were conducted at the beginning and the end of the treatment.

\section{Statistical analyses}

Data were on an intent-to-treat basis, and included all patients who met the entry criteria and contained all the information about the measurements by the statistician who was blind to the experimental condition. Data are presented as means \pm standard deviation. Continuous data were analyzed using the Mann-Whitney $U$-test for nonparametric data. Categorical data were compared the using $\chi^{2}$ test. $P$-values less than 0.05 were considered statistically significant. All statistical analysis was performed using SPSS 16.0 (SPSS Inc, Chicago, IL, USA).

\section{Results Patients' information}

A total of 120 patients were recruited into the study, and were randomly divided into three groups (groups $\mathrm{A}, \mathrm{B}$, and $\mathrm{C}$ ). Forty patients were included in group $A$ at the beginning of the treatment, but only 37 patients (18 males and 19 females) completed this trial. The patients' mean age mean was $31.7 \pm 8.2$ years (range 20-60 years). The mean disease duration was 13.6 \pm 6.2 days (range 9-17 days). Group B included 40 patients at the beginning of the treatment, and 39 patients (19 males and 20 females) completed the trial. The patients mean age was 30.9 \pm 9.1 years (range 21-60 years). Mean disease duration was 14.6 \pm 5.1 days (range 9-21 days). Group C contained 40 patients at the beginning of the treatment, and 38 patients (18 males and 20 females) completed the trial. The patients' mean age was 30.2 \pm 7.8 years (range 19-58 years). Mean dis-

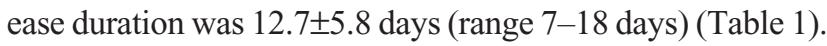
There were no significant differences in age, sex, or disease duration among groups $\mathrm{A}, \mathrm{B}$, and $\mathrm{C}(P>0.05)$ (Table 1).

There was no significant difference in the percentage of patients that completed the trial among the three groups (group A 92.5\%, group B 97.5\%, group C 95.0\%; $P>0.05$ ). In group $\mathrm{A}$, two patients discontinued treatment due to poor efficacy, and one patient initiated withdrawal. In group B, one patient discontinued treatment due to side effects $(5.2 \%$ weight gain in 2 weeks according to our measurements, and unacceptable drowsiness reported by the patient). In group $\mathrm{C}$, one patient discontinued treatment due to side effects $(5.6 \%$ weight gain in three weeks according to our measurements), and one patient withdrew due to abnormal hepatic function (alanine aminotransferase increased to $467 \mathrm{U} / \mathrm{L}$ from $42 \mathrm{U} / \mathrm{L}$ ) (Table 2).

At the end of the treatment, the average dose of sodium valproate was $1.53 \pm 0.22 \mathrm{~g} /$ day in group $\mathrm{A}$, and the average dose of olanzapine was $16.3 \pm 2.1 \mathrm{mg} /$ day in group B.

Table I Baseline characteristics of the patients (mean \pm SD)

\begin{tabular}{llll}
\hline Characteristic & $\begin{array}{l}\text { Combined } \\
\text { therapy }(\mathbf{n = 3 7 )}\end{array}$ & $\begin{array}{l}\text { Olanzapine } \\
\text { monotherapy (n=39) }\end{array}$ & $\begin{array}{l}\text { Valproate } \\
\text { monotherapy }(\mathbf{n}=\mathbf{3 8})\end{array}$ \\
\hline Age, years & $31.7 \pm 8.2$ & $30.9 \pm 9.1$ & $30.2 \pm 7.8$ \\
Female, \% & $51.4 \pm 8.3$ & $51.3 \pm 8.1$ & $52.6 \pm 8.2$ \\
Weight, kg & $68.4 \pm 11.3$ & $69.3 \pm 13.2$ & $72.1 \pm 10.5$ \\
Current course (\%) & & & \\
Manic & $73.0 \pm 7.4$ & $76.9 \pm 6.4$ & $73.7 \pm 7.1$ \\
Mixed & $27.0 \pm 7.4$ & $23.1 \pm 7.0$ & $26.3 \pm 7.0$ \\
Duration of illness, years & $13.6 \pm 6.2$ & $14.6 \pm 5.1$ & $12.7 \pm 5.8$ \\
Baseline YMRS score & $34.25 \pm 6.07$ & $34.55 \pm 7.03$ & $34.39 \pm 9.12$ \\
Baseline CGI-BP scale score & $5.55 \pm 0.76$ & $5.65 \pm 1.09$ & $5.56 \pm 0.86$ \\
Dose of valproate, mg/day & $1.53 \pm 0.22$ & $16.3 \pm 2.1$ & $1.08 \pm 0.45$ \\
\hline
\end{tabular}

Abbreviations: SD, standard deviation; YMRS, Young Mania Rating Scale; CGI-BP, Clinical Global Impression - Bipolar. 
Table 2 Adverse reactions among the three groups

\begin{tabular}{|c|c|c|c|c|c|c|}
\hline \multirow[t]{2}{*}{ Types } & \multicolumn{2}{|c|}{ Group A } & \multicolumn{2}{|c|}{ Group B } & \multicolumn{2}{|c|}{ Group C } \\
\hline & $\bar{n}$ & Ratio (\%) & $n$ & Ratio (\%) & $n$ & Ratio (\%) \\
\hline Weight gain & 21 & $52.5 \pm 8.0$ & 29 & $72.5 \pm 7.1 *$ & 31 & $77.5 \pm 6.7^{*}$ \\
\hline Sleepiness & 6 & $15.0 \pm 5.7$ & 13 & $32.5 \pm 7.5^{*}$ & II & $27.5 \pm 7.1^{*}$ \\
\hline Alopecia & 3 & $7.5 \pm 4.2$ & 0 & 0 & 2 & $5.0 \pm 3.5$ \\
\hline Xerostomia & 3 & $7.5 \pm 4.2$ & 7 & $17.5 \pm 6 . \mid$ & 5 & $12.5 \pm 5.3$ \\
\hline Constipation & 2 & $5.0 \pm 3.5$ & 9 & $22.5 \pm 6.7^{*}$ & 7 & $17.5 \pm 6 . I^{*}$ \\
\hline Nausea & I & $2.5 \pm 2.5$ & 0 & 0 & 0 & 0 \\
\hline Dizziness & 0 & 0 & 6 & $15.0 \pm 5.7^{*}$ & 10 & $25.0 \pm 6.9 *$ \\
\hline Tremor & 0 & 0 & 3 & $7.5 \pm 4.2$ & 2 & $5.0 \pm 3.5$ \\
\hline Abnormal hepatic function & 0 & 0 & 0 & 0 & $\mathrm{I}$ & $2.5 \pm 2.5$ \\
\hline
\end{tabular}

Notes: Group A, sodium valproate monotherapy; Group B, olanzapine monotherapy; Group C, olanzapine and sodium valproate combination. The table shows the different rate of adverse reactions among the three groups. Values presented are mean \pm standard deviation. $* P<0.05$ compared with group $A$.

The average dose of sodium valproate in group $\mathrm{C}$ was $1.08 \pm 0.45 \mathrm{~g} / \mathrm{day}$, which was significantly lower compared with group A $(1.53 \pm 0.22 \mathrm{~g} /$ day, $P<0.05)$. The average dose of olanzapine in group $C$ was $13.1 \pm 3.2 \mathrm{mg} /$ day, which was lower than group $\mathrm{B}$, but there was no significant difference (16.3 $\pm 2.1 \mathrm{mg} /$ day, $P>0.05$ ) (Table 1).

\section{Primary outcomes}

Patients in all three groups showed a significant improvement in YMRS scores as the primary outcome during the course of the treatment. There was no significant difference in the baseline YMRS score among the three groups (group A

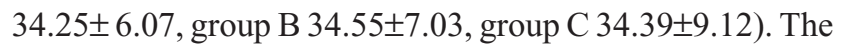
percentage decrease of YMRS score in both groups $\mathrm{B}$ and $\mathrm{C}$ was significantly higher than that in group $\mathrm{A}(P<0.01)$ during the 4-week treatment (Table 3). At the end of the fourth week, patients in group $\mathrm{C}$ showed significantly greater improvement in YMRS score compared with group B $(P<0.01)$. At the end of the fourth week, the YMRS score significantly decreased in group $\mathrm{A}(<75 \%)$ compared with groups $\mathrm{B}$ and $\mathrm{C}(>75 \%$, $P<0.05$ ) (Table 3, Figure 1).

\section{Secondary outcomes}

CGI-BP scale scores were used to assess the secondary outcome in groups A, B, and C. There was no significant difference in baseline CGI-BP scale total scores among the three

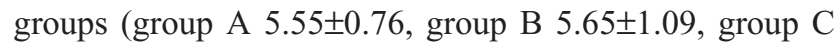
5.56 $\pm 0.86 ; P>0.05$; Table 4). During weeks 1 and 2 , there was no significant difference in baseline CGI-BP scale total scores among the three groups $(P>0.05)$. During weeks 3 and 4 , patients in groups $\mathrm{B}$ and $\mathrm{C}$ showed significant improvement in CGI-BP scale total scores compared with those in group A $(P<0.05)$, and patients in group $\mathrm{C}$ showed significant improvement in CGI-BP scale total scores compared with those in group B $(P<0.01$; Table 4 , Figure 2$)$.

\section{Adverse events}

No statistically significant changes were seen from baseline in extrapyramidal symptoms on the Simpson-Angus Scale. Rates of adverse events, including weight gain, sleepiness, constipation, and dizziness, were more frequently reported in groups $\mathrm{B}$ and $\mathrm{C}$ than in group $\mathrm{A}(P<0.05$; Table 2$)$.

\section{Discussion}

In this double-blind, randomized controlled study, we found that combination therapy of olanzapine-sodium valproate had significantly better efficacy in managing bipolar I manic episodes than valproate monotherapy, assessed by YMRS and CGI-BP scale scores, in line with previous studies. Furthermore, we also found that the combination therapy had an advantage over olanzapine monotherapy in efficacy, which was not shown in previous randomized controlled trials.

Table 3 Percentage decrease in Young Mania Rating Scale score among the three groups

\begin{tabular}{lllll}
\hline Group & First week & Second week & Third week & Fourth week \\
\hline A & $12.45 \pm 7.03$ & $24.37 \pm 6.31$ & $41.38 \pm 4.52$ & $55.11 \pm 5.72$ \\
B & $19.15 \pm 6.48^{*}$ & $40.24 \pm 11.76^{*}$ & $60.03 \pm 15.44^{*}$ & $75.19 \pm 15.08^{*}$ \\
C & $21.80 \pm 11.72^{*}$ & $46.46 \pm 16.36^{*}$ & $68.36 \pm 13.03^{*}$ & $86.47 \pm 8.85^{*, \Lambda}$ \\
F-value & 6.062 & 17.354 & 25.875 & 42.408 \\
P-value & 0.004 & 0 & 0 & 0 \\
\hline
\end{tabular}

Notes: Group A, sodium valproate monotherapy; Group B, olanzapine monotherapy; Group C, olanzapine and sodium valproate combination. The table shows the decrease in Young Mania Rating Scale among the three groups. Values presented are mean \pm standard deviation. $* P<0.0$ I versus group $A$; $\wedge p<0.0$ I versus group $B$. 


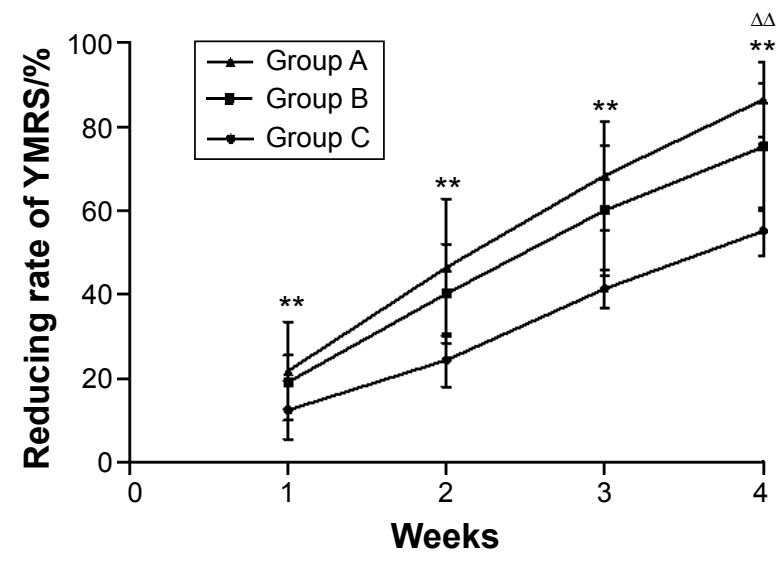

Figure I The rate of Young Mania Rating Scale (YMRS) score reduction changed during the treatment.

Notes: Group A, sodium valproate monotherapy; Group B, olanzapine monotherapy; Group C, olanzapine and sodium valproate combination. Reducing rate of Young Mania Rating Scale (YMRS) score among the three groups. Values presented are mean \pm standard deviation. ${ }^{* * P}<0.0$ I group $B$ or group $C$ versus Group $A ;{ }^{\Delta} P<0.01$ group $C$ versus group $B$. Data show the percentage decrease of $Y M R S$ in group $A$ $(n=37)$, group $B(n=39)$, and group $C(n=38)$.

Patients on olanzapine monotherapy showed significantly greater improvement of outcome than patients on sodium valproate monotherapy. There were no significant differences in extrapyramidal symptoms among patients on different treatments, but the number of adverse events was higher in patients receiving olanzapine monotherapy or combined therapy.

In the present study, the enrolled subjects were in their first acute manic episode without prior treatment with valproate or olanzapine, which was different from the previous studies, ${ }^{3,11,12}$ while the dosage-administration method and internals of follow-up interview and assessment methods in this study were similar to previous studies, which proved to be acceptable to subjects and reasonable for the trial.

We also found that after 1 week of treatment, the percentage decrease in YMRS scores was significantly higher in patients receiving olanzapine monotherapy than in patients receiving valproate monotherapy. Moreover, after 3 weeks of treatment, CGI-BP scale total scores were significantly lower in patients receiving olanzapine than in patients receiving valproate. These findings suggest that olanzapine monotherapy has a faster effect and is more effective in treating bipolar I mania than valproate monotherapy. Consistent with this, an open-label, 8-week trial of olanzapine or valproate for the treatment of bipolar manic relapse in Italian adult patients has shown that patients receiving olanzapine had significant improvement in YMRS scores after 1 week of treatment. ${ }^{11}$ Furthermore, another prospective open-label trial of olanzapine monotherapy and olanzapine combination therapy in Europeans with bipolar mania disorder found that olanzapine treatment significantly reduced CGI-BP scale scores after 1 week. ${ }^{15}$ Taken together, these studies suggest that olanzapine can produce efficacy in managing bipolar I manic episodes as early as 1 week after the start of treatment.

Interestingly, the present study also found that the combination therapy of valproate with olanzapine showed similar efficacy by YMRS score within 3 weeks of the treatment compared with olanzapine monotherapy, suggesting that valproate had no significant efficacy after short-term treatment ( 3 weeks). Consistent with our findings, it has been reported that after 3 weeks of olanzapine but not valproate treatment, patients with bipolar I manic episodes showed significantly greater improvement in YMRS scores than controls. ${ }^{16}$ Furthermore, a 47-week trial for the treatment of bipolar I mania has shown that the average onset of efficacy is 14 days for olanzapine and 62 days for valproate. ${ }^{17}$ Our study found that the percentage decrease in YMRS scores in the group on valproate monotherapy reached over $50 \%$ only at the end of the fourth week, suggesting that valproate needed 3 weeks to produce efficacy for the treatment of bipolar I mania. Therefore, the use of olanzapine monotherapy provides superior efficacy in managing bipolar I manic episodes.

In addition, we found that adverse events, including weight gain, sleepiness, dizziness, and constipation, occurred more frequently in patients receiving olanzapine therapy. This finding was in line with previous reports showing that the use of atypical antipsychotics increased the incidence of weight gain ${ }^{18}$ and olanzapine in combination with valproate had a higher incidence of dizziness than valproate monotherapy. ${ }^{3}$ Importantly, in the present study, olanzapine monotherapy also caused dizziness more frequently

Table 4 The Clinical Global Impression - Bipolar scale total score among the three groups

\begin{tabular}{llllll}
\hline Group & Baseline & First week & Second week & Third week & Fourth week \\
\hline A & $5.55 \pm 0.76$ & $4.80 \pm 0.83$ & $3.95 \pm 0.51$ & $3.40 \pm 0.60$ & $2.50 \pm 0.51$ \\
B & $5.65 \pm 1.09$ & $4.65 \pm 1.04$ & $3.50 \pm 1.19$ & $2.60 \pm 1.19 *$ & $1.80 \pm 1.06 *$ \\
C & $5.56 \pm 0.86$ & $4.39 \pm 0.98$ & $3.22 \pm 0.88$ & $1.89 \pm 0.47^{* * . \Lambda}$ & $1.06 \pm 0.24 * * \wedge \Lambda$ \\
F-value & 0.075 & 0.895 & 3.151 & 15.984 & 20.028 \\
P-value & 0.928 & 0.415 & 0.051 & 0 & 0 \\
\hline
\end{tabular}

Notes: Group A, sodium valproate monotherapy; Group B, olanzapine monotherapy; Group C, olanzapine and sodium valproate combination. ${ }^{* P}<0.05$, $* * P<0.01$ compared with group $A ; \wedge P<0.05, \wedge \wedge P<0.01$ compared with group $B$. Values presented are mean \pm standard deviation. 


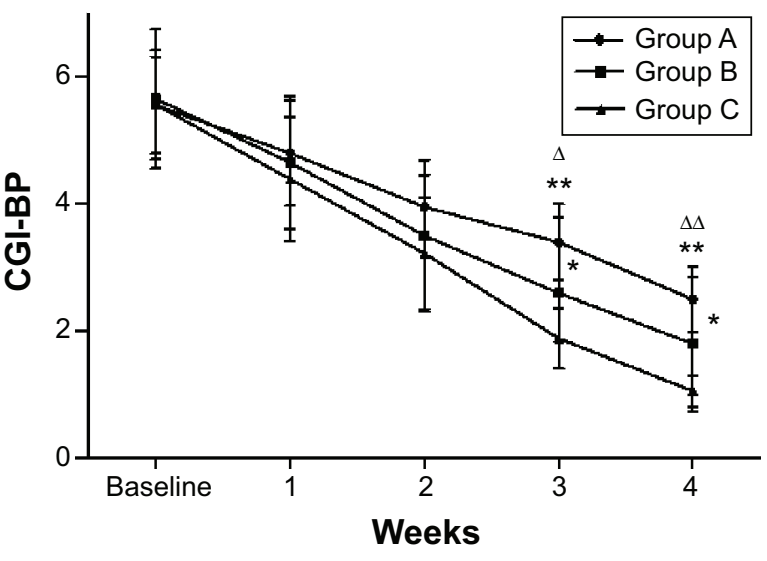

Figure 2 Clinical Global Impression - Bipolar (CGI-BP) scale scores changed during the treatment.

Notes: Group A, sodium valproate monotherapy; Group B, olanzapine monotherapy; Group C, olanzapine and sodium valproate combination. The change of Clinical Global Impression - Bipolar (CGI-BP) scale scores among the three group. Values presented are mean \pm standard deviation. $* P<0.05$, $* * P<0.01$ group $B$ or group $C$ versus group $A ;{ }^{\Delta P}<0.05, \triangle P P<0.01$ group $C$ versus group $B$. Data show the CGI-BP scale scores of group $A(n=37)$, group $B(n=39)$, and group $C(n=38)$.

than valproate monotherapy, suggesting that there is no clear synergism between olanzapine and valproate in this adverse effect. However, the exact mechanisms underlying olanzapine-induced side effects remain unclear. Recent studies have shown that the gastric ghrelin-signaling system ${ }^{19}$ and extrahepatic insulin resistance ${ }^{20}$ may contribute to olanzapine-induced side effects. In addition, there was no significant difference in baseline extrapyramidal reactions after treatment, suggesting that olanzapine did not cause extrapyramidal side effects.

Despite the sound results, the present study has some limitations. Firstly, this study did not include a placebo control group to confirm the efficacy of olanzapine or sodium valproate monotherapy, which was due to the concern that patients with manic episodes should be actively controlled with drugs. Secondly, the present study recruited only 120 patients with bipolar I manic episodes ( $\mathrm{n}=40$ for each group). Although we found a significant difference in the efficacy of the medication among groups, the sample size of this study was small. Clinical trials with a larger sample size are required to confirm the results of this study in the future.

\section{Conclusion}

In this study, we found that in patients with bipolar I manic episodes, the combination therapy of olanzapine and sodium valproate had significantly better efficacy than valproate or olanzapine monotherapy. Although patients on olanzapine therapy experienced more adverse side events, none of these side effects seemed to be life-threatening. Therefore, our work presents a safe but novel approach for olanzapine-valproate combination treatment versus olanzapine or valproate monotherapy to improve clinical outcome in managing bipolar I manic episodes.

\section{Acknowledgments}

This trial was registered with the Chinese Clinical Trial Registry (ChiCTR-TRC-13003554). This work was supported by grants from the Health Department Foundation of Zhejiang Province (2011KYA073), the Natural Science Foundation of Zhejiang Province (Y2100294), and the Science and Technology Department Foundation of Zhejiang Province (2010C33038). We thank Professor Xiaofei Zhang, Department of Statistics, Second Affiliated Hospital, Zhejiang University School of Medicine, for providing statistical analysis help.

\section{Author contributions}

All authors contributed toward data analysis, drafting and revising the paper and agree to be accountable for all aspects of the work.

\section{Disclosure}

The authors report no conflicts of interest in this work.

\section{References}

1. Merikangas KR, Akiskal HS, Angst J, et al. Lifetime and 12-month prevalence of bipolar spectrum disorder in the National Comorbidity Survey replication. Arch Gen Psychiatry. 2007;64(5):543-552.

2. Merikangas KR, Jin R, He JP, et al. Prevalence and correlates of bipolar spectrum disorder in the World Mental Health Survey Initiative. Arch Gen Psychiatry. 2011;68(3):241-251.

3. Tohen M, Chengappa KN, Suppes T, et al. Efficacy of olanzapine in combination with valproate or lithium in the treatment of mania in patients partially nonresponsive to valproate or lithium monotherapy. Arch Gen Psychiatry. 2002;59(1):62-69.

4. Hoge SK, Appelbaum PS, Lawlor T, et al. A prospective, multicenter study of patients' refusal of antipsychotic medication. Arch Gen Psychiatry. 1990;47(10):949-956.

5. Sumiyoshi T, Higuchi Y, Uehara T. Neural basis for the ability of atypical antipsychotic drugs to improve cognition in schizophrenia. Front Behav Neurosci. 2013;7:140.

6. Tohen M, Sanger TM, McElroy SL, et al. Olanzapine versus placebo in the treatment of acute mania. Olanzapine HGEH Study Group. Am J Psychiatry. 1999;156(5):702-709.

7. Tohen M, Jacobs TG, Grundy SL, et al. Efficacy of olanzapine in acute bipolar mania: a double-blind, placebo-controlled study. The Olanzipine HGGW Study Group. Arch Gen Psychiatry. 2000;57(9): 841-849.

8. Zarate CA Jr, Narendran R, Tohen M, et al. Clinical predictors of acute response with olanzapine in psychotic mood disorders. J Clin Psychiatry. 1998;59(1):24-28.

9. Tohen M, Baker RW, Altshuler LL, et al. Olanzapine versus divalproex in the treatment of acute mania. Am J Psychiatry. 2002;159(6):1011-1017.

10. Zajecka JM, Weisler R, Sachs G, Swann AC, Wozniak P, Sommerville KW. A comparison of the efficacy, safety, and tolerability of divalproex sodium and olanzapine in the treatment of bipolar disorder. J Clin Psychiatry. 2002;63(12):1148-1155. 
11. Maina G, Albert U, Salvi V, Mancini M, Bogetto F. Valproate or olanzapine add-on to lithium: an 8-week, randomized, open-label study in Italian patients with a manic relapse. J Affect Disord. 2007;99(1-3):247-251.

12. Baker RW, Brown E, Akiskal HS, et al. Efficacy of olanzapine combined with valproate or lithium in the treatment of dysphoric mania. Br J Psychiatry. 2004;185:472-478.

13. Gardner DM. The addition of olanzapine to valproate or lithium for acute manic or mixed bipolar episodes reduced manic symptoms. Evid Based Ment Health. 2002;5(3):89.

14. Cazorla P, Zhao J, Mackle M, Szegedi A. Asenapine effects on individual Young Mania Rating Scale items in bipolar disorder patients with acute manic or mixed episodes: a pooled analysis. Neuropsychiatr Dis Treat. 2013;9:409-413.

15. Vieta E, Panicali F, Goetz I, Reed C, Comes M, Tohen M. Olanzapine monotherapy and olanzapine combination therapy in the treatment of mania: 12-week results from the European Mania in Bipolar Longitudinal Evaluation of Medication (EMBLEM) observational study. $J$ Affect Disord. 2008;106(1-2):63-72.
16. Tohen M, Vieta E, Goodwin GM, et al. Olanzapine versus divalproex versus placebo in the treatment of mild to moderate mania: a randomized, 12-week, double-blind study. J Clin Psychiatry. 2008;69(11): 1776-1789.

17. Tohen M, Ketter TA, Zarate CA, et al. Olanzapine versus divalproex sodium for the treatment of acute mania and maintenance of remission: a 47-week study. Am J Psychiatry. 2003;160(7):1263-1271.

18. Lieberman JA, Stroup TS, McEvoy JP, et al. Effectiveness of antipsychotic drugs in patients with chronic schizophrenia. $N$ Engl $J$ Med. 2005;353(12):1209-1223.

19. Zhang Q, Deng C, Huang XF. The role of ghrelin signalling in second-generation antipsychotic-induced weight gain. Psychoneuroendocrinology. 2013;38(11):2423-2438.

20. Girault EM, Alkemade A, Foppen E, Ackermans MT, Fliers E, Kalsbeek A. Acute peripheral but not central administration of olanzapine induces hyperglycemia associated with hepatic and extra-hepatic insulin resistance. PloS One. 2012;7(8):e43244.
Neuropsychiatric Disease and Treatment

\section{Publish your work in this journal}

Neuropsychiatric Disease and Treatment is an international, peerreviewed journal of clinical therapeutics and pharmacology focusing on concise rapid reporting of clinical or pre-clinical studies on a range of neuropsychiatric and neurological disorders. This journal is indexed on PubMed Central, the 'PsycINFO' database and CAS,

\section{Dovepress}

and is the official journal of The International Neuropsychiatric Association (INA). The manuscript management system is completely online and includes a very quick and fair peer-review system, which is all easy to use. Visit http://www.dovepress.com/testimonials.php to read real quotes from published authors. 